\title{
Multiple encoding of word attributes in memory
}

\author{
DELOS D. WICKENS and RUTH E. DALEZMAN \\ The Ohio State University, Columbus, Ohio 45210
}

and

\section{F. THOMAS EGGEMEIER \\ Air Force Human Resources Laboratory, Wright-Patterson Air Force Base, Dayton, Ohio 45439}

\begin{abstract}
Two experiments employing the release from PI method were conducted to determine whether degree of release could be predicted by the amount of denotative attribute overlap between the control and experimental groups. This theory was very appropriate for predicting the results of the first experiment, but was less successful in the second unless a connotative attribute is also introduced. It is suggested that this class of attribute should be included in semantic memory conceptualization.
\end{abstract}

One approach to modern semantic memory theory assumes that the perception of the memory for verbal information is based upon the encoding of different aspects or attributes of the information-a word-within various dimensions. These attributal dimensions could be orthogonal, as in the case of connotative meaning in the Osgood Semantic Differential (Osgood, Suci, \& Tannenbaum, 1957); unspecified in their interrelationships, as in other theories (Bower, 1967; Shaeffer \& Wallace, 1970; Underwood, 1969; Wickens, 1970); or they could be related to some hierarchical structure (Collins \& Quillian, 1969). All of these classes of systems would state that many words share certain attributes in common, but will differ from each other on one or more attributes.

The purpose of the present research was to investigate the attribute view of word meaning through the medium of the release from proactive inhibition (PI) technique. In the release from PI technique (Wickens, 1970), the experimenter gives memory tests for triads of words of the same conceptual class for three trials and then shifts to a triad of words drawn from a different conceptual class on the fourth trial. Under many, but not all, types of shifts, performance on the fourth trial will show a marked increment.

The authors' basic interpretation as to why one obtains release from PI is that it arises as a consequence of a decrement in interference during the retention test. This decrement will occur if a subsequent set of items is drawn from a psychologically different class than were the previous sets. It is further assumed that the memory performance is a reflection of how the materials were

This research was supported by NSF Grant GB 33680 and NIMH Grant MH 08526-11. Requests for reprints should be sent to: Delos D. Wickens, Department of Psychology, 1945 North High Street, The Ohio State University, Columbus, Ohio 43210. encoded in the first place, for it seems certain that if differences are to have an effect in memory these differences must have existed in some form at the perceptual stage so as to be mapped into the memory process. All changes in materials do not show the release effect, or to the same degree (Wickens, 1970), and a plausible interpretation of this finding is that the magnitude of the release is a function of the psychological distance between the classes to which the items belong. The closer they are, the less the decrement in interference.

This statement at first blush sounds too general, for there are certain sorts of class shifts which are relatively easy to identify, as are verbs to nouns, past to present tense, or singular to plural, but they produce essentially no release. One might assume, however, that most of us develop strong biases to encode words primarily along a semantic dimension because this dimension is essential if communication is to occur. When, in this demanding memory task, the subjects are told that they will see and will be asked to recall words, this description of the task may increase an already dominant set for semantic encoding to the exclusion of some other possible types of encoding.

This interpretation leads to the conclusion that the magnitude of semantic distance would be reflected in degree of release from PI. The problem, of course, is to find a system which would predict semantic differences before beginning the experiment. A potential solution to this problem is to make a prediction based upon the amount of overlap of denotative attributes between groups and to vary the amount of overlap among groups predicting varying amounts of release from PI as a function of the amount of overlap. The two experiments to be reported were done in an effort to test the usefulness of this system of prediction in the release from PI situation. Specifically, the experiments were designed to determine if different amounts of release would 
occur for sets of words which differed in the number of obvious denotative common attributes, the numbers being two, one, and zero.

In Experiment 1. each of the five groups received word triads draun from the category of fruits on Trial 4: the groups differed from each other in the ategory of the words employed on the first three trials. Those categories were: professions. flowers. meats. and vegetables. Of course. the control group received fruit examples on all four trials. Note that regetables have two major attributes in common with fruits. Both of them are edible and both grow from the ground. Meats, of course, are edible, but they do not grow from the ground. Flowers typically grow from the ground, but are not edible. Professions share neither of these characteristics. Thus, one has sets of words which overlap with fruits in their major attributes in two, one, or zero characteristics. They therefore might be expected to produce differential magnitude effects in the release from PI situation. being ordered inversely in terms of the shared attributes.

The second experiment used the same logic but a different set of categories. On the fourth trial, all groups received a triad of words from the category of consumable nonalcoholic liquids. As in the first experiment. the four experimental groups differed in the category employed during the first three trials. One group received words from the class of alcoholic beverages, a class which shows two attributes. consumability and liquidity. in common with the target trial class. Another group received liquids. The third group also overlapped the Trial 4 class in only one category and they received words from a consumable, but not liquid, class-meats. Professions once again served as the nonoverlapping class for the final experimental group.

\section{METHOD}

\section{Subjects}

There were 32 elementary psychology student subjects in each of the five groups in the two experiments. They had selected this experiment to meet a course requirement. They were assigned to groups in rotating order as they appeared in the laboratory.

\section{Materials}

In Experiment 1. the following triads were used: Fruit-banana. peach. apple: pear. grape. cherry: lemon. plum. apricot; pineapple. lime. melon. Vegetuble-onion. spinach. carrot: asparagus, potato, broccoli; celery, radish, bean. Flowers-pansy. lilac, daisies; violet, orchids, tulip; honeysuckle, clover, poppy. Meats-salami. chops. roast; ribs. mutton. sausage; hamburger. veal, bacon.

In Experiment 2, the following triads were employed: Nonalcoholic beverages-lemonade, shake, cocoa; cider, pop. juice: soda. orangeade, cream; Kool-Aid, Pepsi, tea. Alcoholic beverages-champagne, brandy, vodka; bourbon, martini, scotch: gin. daiquiri. rum. Nonconsumable liquids-gasoline. ink. ammonia: acid. kerosene. bleach; antifreeze, perfume. mercury. The triads for meats and professions were the same as those in Experiment 1.
The particular words chosen were drawn from the Battig and Montague (1969) norms, with the exception of meats and liquids, which are not included by them. Words for these categories were obtained by asking about 50 college students to generate examples of the class-meats and liquids. An effort was made to equate the triads in mean word frequency using the Kučera and Francis (1967) norms.

\section{Procedure}

The materials were presented by a Carousel projector at an intertrial interval of $30 \mathrm{sec}$. A $2 \mathrm{-sec}$ warning asterisk was followed by a word triad for $2 \mathrm{sec}$ and was replaced by a threc-digit number from which the subject counted backwards by threes for $18 \mathrm{sec}$. A question mark signaled the request for recall, and it remained on until the warning signal for the next trial occurred. giving $8 \mathrm{sec}$ for recall. Both experiments followed the same procedure and were conducted in successive quarters. Responses were scored by giving 1 point for each word recalled and an additional point if all three words were recalled in the presentation order. The triads were counterbalanced across trials and subjects.

\section{RESULTS}

The results of the two experiments are summarized graphically in Figures 1 and 2. The statistical methods of analysis employed were, first, an analysis of variance across all four trials including all groups and subsequent Tukey A tests at the various trial points.

In Experiment 1. the Anova showed a significant trials effect $[F(3.345)=89.79, p<.01]$, a significant Trials by Groups interaction $\mid F(12.345)=4.98$. $p<.01]$. The MSe was 1.18. The subsequent Tukey A tests were set at the .05 level, and this level was not reached on any of the first three trials. On Trial 4. the following ordering was obtained:

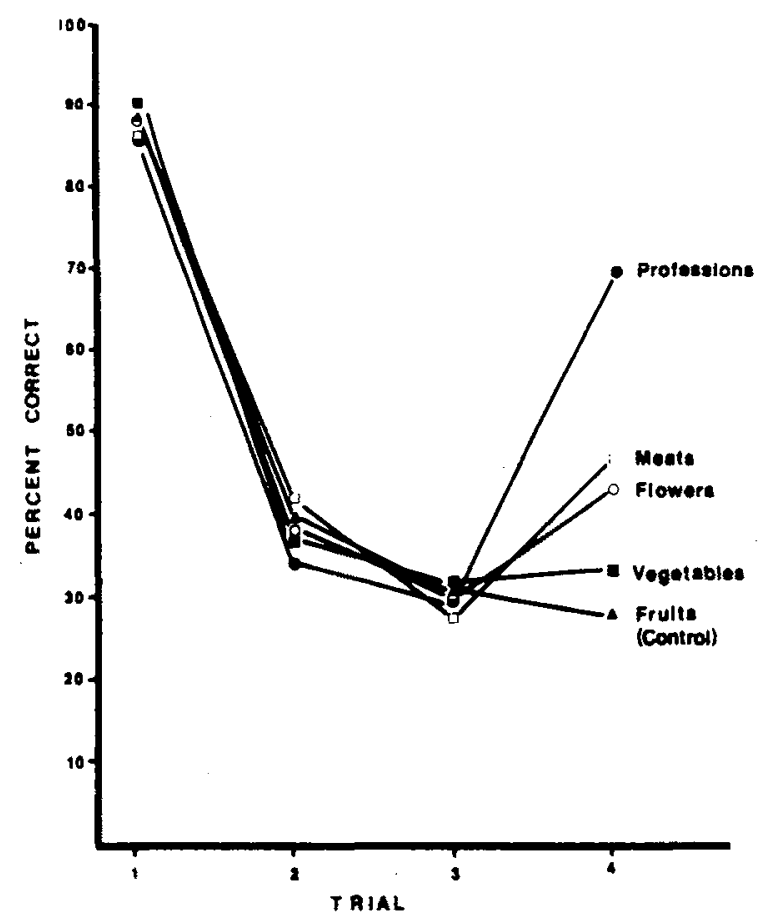

Figure 1. Performance across trials as a function of described shifts in Experiment 1. 


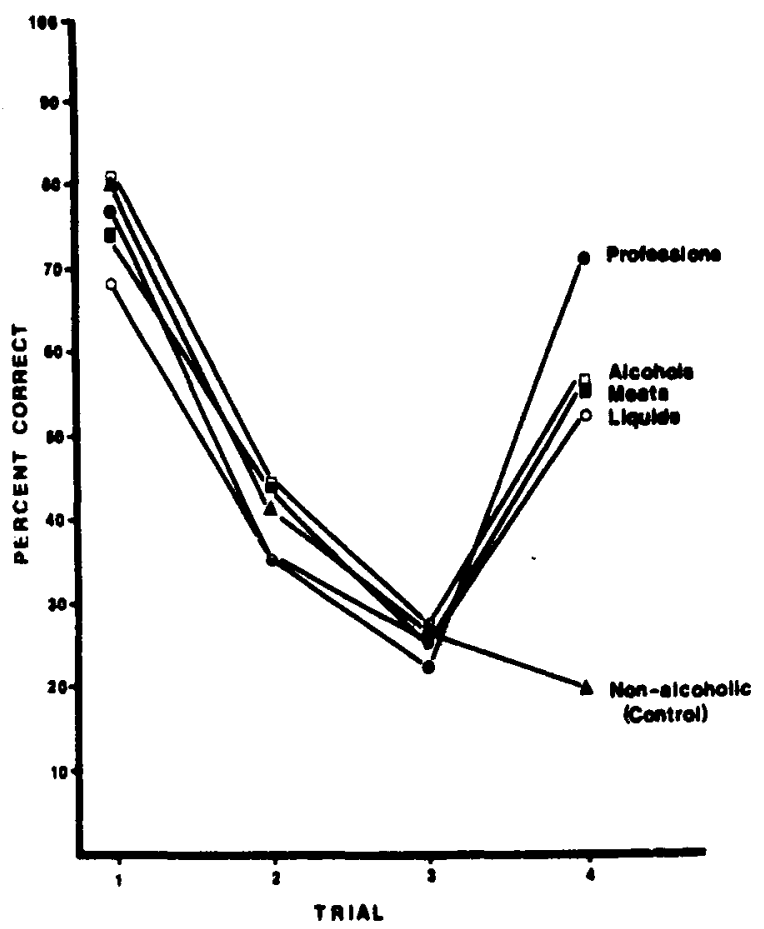

Figure 2. Performance across trials as a function of described shifts in Experiment 2.

professions $>$ flowers $=$ meats $>$ vegetables $>$ fruits.

Experiment 2 was treated in the same fashion. The Anova produced a trials effect of $F(3,345)=95.0$, $p<.01$, and a Trials by Group effect of $F(12,345)=$ $4.68, \mathrm{p}<.01$. The MSe was .94. The subsequent Tukey A tests at the .05 level were not significant on any of the first three trials, but gave the following ordering on Trial 4: professions $>$ alcoholic beverages $=$ meats $=$ liquids $>$ nonalcoholic beverages. All of the significant and nonsignificant trends are fairly obvious from an inspection of the two figures.

\section{DISCUSSION}

The results of Experiment 1 are very nicely predicted by the attribute overlap theory, for the degree of release from PI is inversely related to the number of common attributes. The vegetable group which overlaps fruits in two attributes-consumability and growing from the ground-obtains the least, although a significant, amount of release from PI. Meats overlap on a single attribute-consumability, as do flowers (grown from the ground), and these two conditions give more, but essentially equal, release. The professions group, which has no obvious overlap with fruits, produces the most and a very high amount of release.

In Experiment 2, however, this simple theory is no longer predictive of the results. Although all experimental groups show a release from PI, and professions produced the most. alcoholic beverages which overlaps nonalcoholic beverages in two attributes-consumable and liquidity-does not differ from the single-attribute groups. Actually, it is slightly, but not significantly, higher than those two groups. The attribute overlap theory does not seem to possess sufficient generality to be completely acceptable.

There is, however, a post hoc explanation which might salvage the theory and account for the higher than expected release from PI for the alcohol group. This explanation assumes that a connotative attribute, namely evaluative (good-bad), operates for the alcohol group but does not do so for the other groups. We may safely assume that for a number of the subjects in the experiment the alcoholic beverages that were presented would be conceived of as being bad and that this attribute would be involved in their encoding of the three initial triads. On the fourth trial, they experience a triad naming nonalcoholic beverages which we may safely assume would be rated positively by all of the subjects. Thus, for many subjects in the alcohol group, the fourth trial presentation involves a shift in two attributes, the denotive attribute of alcohol as a chemical and also the connotative one of location on the evaluation dimension. It is known (Wickens \& Clark, 1968) that a shift from one to the other end of this dimension is associated with a considerable amount of release from PI. This interpretation converts the alcohol group to a shift of two attributes rather than a single attribute change as was assumed in the beginning of the experiment. One can consider that the words of the other experimental groups do not have a negative evaluation, as does alcohol, for at least some subjects. This interpretation, and it seems to be a plausible one, would account for the alcohol group's showing the same release effect as do the other two denotative two-attribute shift groups. All of these groups fall below the zero-attribute professions group. This interpretation requires that semantic memory must include connotative as well as denotative states in the description of the structure of that memory.

It seems pertinent at this time to compare the results of the release from PI technique with those of another method. In an experiment by Collen, Wickens, and Daniele (1975), college populations were asked to sort the category names of the Battig and Montague norms (with the addition of meats) into as many or as few piles as they wished. The percentages of sorts with the category of fruits for the classes used in Experiment 1 were: professions ( 0 ), flowers (25), meats (64), and vegetables (90). These percentages are in inverse ranking of the degree of release from PI for that experiment. For Experiment 2, however, the sorting percentages with nonalcoholic beverages were: professions (1), meats (35), and alcohols (84). Liquids of the type exemplified in this experiment were not included in the category sorts. It will be noted that in the sorting tasks the two potable liquids are classified together 
with high frequency, nearly as high as are fruits and vegetables of Experiment 1 . Thus, the sorting data do not predict the results of Experiment 2 as they do for Experiment 1.

It might be argued that the release from PI data may be too variable to expect accuracy in prediction, but there is evidence of considerable stability between the two experiments. The control groups' mean correct scores on Trial 4 were .83 and .75 out of a possible 4.0 in the two experiments. Meats and professions were used in both experiments, the Trial 4 performance of the professions group was 2.83 in both experiments, and the scores for the meats groups were 2.21 and 2.25 in the two experiments. This consistency across two experiments suggests that the rank orderings of the groups are likely to be quite reliable.

If one considers that the organization of semantic or lexical memory (Collins \& Quillian, 1969) is being tapped in both classes of experiments, the sorting and the release from PI, it is apparent that the organization is not represented as being the same when one method is used as compared with the other in Experiment 2, and the question is why this should be so. The sorting method allows the subject ample time to consider and reconsider his classification judgments, and the directions of the task may lead to the adoption of a denotative set and the exclusion of connotative evaluations. No clear preparatory sets on the analysis of word meaning are given in the release from PI technique, and perhaps the subjects use the type of analysis we use when we react to words in the usual conversational or reading situation; situations in which we are usually not set for encoding by one class of attributes alone. Often. for example, a speaker will say "and I use the word in a nonpejorative sense," thus giving a direction to the listeners to encode the word only along denotative dimensions. The warning expresses the speaker's implicit assumption that the organization of semantic memory includes the connotative as well as denotative set of attributes, but that a particular set or control process can limit the nature of the recipients's encoding process.

Parenthetically, it should be mentioned that a tree diagram solution was attempted under the assumption that the amount of release should be a function of the number of nodes intervening between control items and shift items. Unfortunately, the solution was not unique for the types of categories employed, and neither of the diagrams predicted perfectly using the additive node concept. A multidimensional scaling method of distance might prove more effective for predicting in this type of situation, provided, of course, the directions for the task do not limit the subjects to any one class of attributes, the denotative or the connotative.

On the basis of the present research, it is suggested that a word is not stored as a single entity in semantic memory, but as marked locations on two major dimensions, the denotative and the connotative. Note, for example, that one can generate words that connote good or bad as well as words that denote geographical locations. Within each of these major dimensions, there are independent subdimensions which locate the attributes of the word. Any given verbal input is stored on various loci of all of these dimensions, although the word itself may end up as a multidimensional vector. Proactive inhibition builds up for words which globally we called similar. It does so because the same locus is activated by the successive words of a similar sort-defined by their salient attributes-and, for a certain period of time, the pathways to the other, the less salient and uniquely defining attributes of these words, can be easily excited by another word containing the salient attributes. The result is a competition for direction of outlet and blocking of the response, or, in some cases, a previous word pathway is activated leading to an intrusion in recall. Release from PI occurs when one or several salient loci of attributes are changed and the significant earlier ones are no longer involved in identifying the new words. Hence there is no competition produced by words just previously experienced.

One is tempted to assume that, so far as the nervous system is concerned, the attributes have different physical locations and activate different cells or cluster of cells, but too little is known about the central nervous systems at this detailed level to press the point.

\section{REFERENCES}

Batrig, W. F., \& Montague, W. E. Category items for verbal items in 56 categories: A replication and extension of the Connecticut category norms. Journal of Experimental Psychology Monographs, 1969, 80(3 Pt. 2).

BowER, G. H. A multicomponent theory of the memory trace. In K. W. Spence \& J. T. Spence (Eds.). The psychology of learning and motivation (Vol. 1). New York: Academic Press, 1967. Pp. 229-325.

Collen, A., Wickens, D. D., \& Daniele, L. The interrelationship of taxonomic categories. Journal of Experimental Psychology, 1975, 1. 629-633.

Collins, A. M., \& Qullian, M. R. Retrieval time from semantic memory. Journal of Verbal Learning and Verbal Behavior, 1969. 8. 240-248.

KuČERA, H., \& Francis, W. N. Computational analysis of present-day American English. Providence, R.I: Brown University Press, 1967.

Osgood, C. E., Suci, G. J., \& Tannenbaum, P. H. The measurement of meaning. Urbana: University of Illinois Press, 1957.

Shaeffer, B., \& Wallace, R. The comparison of word meanings. Journal of Experimental Psychology, 1970, 86, 144-152.

UNDERWOOD, B. J. Attributes of memory. Psychological Review, $1969,76.559-573$.

Wickens, D. D. Encoding categories of words: An empirical approach to meaning. Psychological Review, 1970, 77, 1-15.

Wickens, D. D., \& Clark, S. E. Osgood dimensions as an encoding category in short-term memory. Journal of Experimental Psychology. 1968, 78. 580-584.

(Received for publication July 20, 1975; revision accepted September 8. 1975.) 